\title{
Efectividad de un programa de capacitación en diseño de prótesis parcial removible en estudiantes de odontología de internado estomatológico
}

\author{
Effectiveness of Training program in removable partial prosthesis design in dental \\ students of the stomatological internship
}

\author{
Caro Magni Rosa ${ }^{1 a}$, Janet-Ofelia Guevara Canales ${ }^{2 b}$, Rafael Morales Vadillo ${ }^{2 c}$, Nelly Tanaka Torres ${ }^{1 b}$, \\ Jimmy Aliaga Fernández ${ }^{1 a}$
}

\section{RESUMEN}

Objetivo: El objetivo del presente estudio fue determinar la efectividad de un programa de capacitación en diseño de PPR en estudiantes de la asignatura de Internado Estomatológico de la Facultad de Odontología de la Universidad de San Martin de Porres. Materiales y Métodos: La muestra estuvo conformada por 70 estudiantes, se tomó un cuestionario cognitivo de 20 preguntas y una evaluación procedimental a cada uno en dos momentos diferentes, antes y después de realizar un programa de capacitación en diseño de PPR. Resultados: Se encontró que existen diferencias estadísticamente significativas $(p<0.001)$ entre las evaluaciones iniciales y finales de las competencias cognitivas y procedimentales en diseño de PPR. Conclusiones: Se concluye que el programa de capacitación en diseño de PPR es efectivo.

PALABRAS CLAVE: Programa de Capacitación; Diseño; Prótesis Parcial Removible.(Fuente: DeCS BIREME)

\section{ABSTRACT}

Objective: The objective of the present study was to determine the effectiveness of a training program in PPR design in students of the Stomatological Internship course at Faculty of Dentistry of the University of San Martin de Porres. Materials and Methods: The sample consisted of 70 students, a 20-question cognitive questionnaire and a procedural evaluation were taken for each one at 2 different moments, before and after carrying out a training program in PPR design. Results: It was found that there are statistically significant differences $(p<0.001)$ between the initial and final evaluations of the cognitive and procedural competences in the PPR design. Conclusions: It is concluded that the PRP design training program is effective

KEY WORDS: Training Program; Design; Removable Partial Denture. (Source: MeSH NLM)

${ }^{1}$ Universidad Nacional Mayor de San Marcos. Especialidad Rehabilitación Oral. Lima, Perú

${ }^{2}$ Universidad de San Martin de Porres. Maestría en Odontología - Lima, Perú

${ }^{a}$ Docente Pos Grado

${ }^{b}$ Coordinadora, Docente de Posgrado

${ }^{\mathrm{C}}$ Director del Instituto de Investigación, Docente de Posgrado

Correspondencia:

Rosa Soledad Caro Magni

Correo electrónico: rosisol84@hotmail.com
Este es un artículo Open Access distribuido bajo la licencia Creative Commons Atribución-NoComercialCompartirlgual 4.0

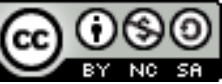

Citar como: Caro Magni R, Guevara Canales JO, Morales Vadillo R, Tanaka Torres N, Aliaga Fernández J. KIRU. 2020; 17(4): 184-193.https://doi.org/10.24265/kiru.2020.v17n4. 


\section{INTRODUCCIÓN}

Un programa de capacitación es un conjunto de actividades que están estructuradas y sistematizadas para cumplir las metas propuestas. Ayudan a potenciar objetivos y planificar las actividades. La evaluación del estudiante debe ser constante, integral e innovadora dando como resultado profesionales competentes con conocimientos de los procesos cognitivos, procedimentales y actitudinales ${ }^{(1)}$.

Una competencia entonces es un conjunto de comportamientos y habilidades que permiten desempeñar adecuadamente una actividad o tarea ${ }^{(2)}$. En odontología, es definida como el comportamiento de los egresados de las facultades para insertarse al mundo laboral. Este comportamiento incluye conocimientos, actitudes y prácticas frente a los requerimientos del paciente ${ }^{(3)}$

La prótesis parcial removible (PPR) es un aditamento removible que se encarga de reemplazar los dientes y tejidos perdidos estabilizando la salud bucal del paciente ${ }^{(4-7)}$. La reposición de los dientes perdidos mediante la PPR permitirá restablecer la función del sistema estomatognático ${ }^{(8,9)}$, para ello es necesario conocer el diagnóstico, pronóstico y plan de tratamiento del paciente edéntulo parcial.

El diseño de la PPR es la esquematización de la estructura metálica de la futura PPR, se realiza diseñando un trazo sobre un modelo de yeso paralelizado previamente. La preparación de la boca es una fase importante dentro del diseño de la PPR, ya que sin ella diversos tratamientos protésicos fracasarían. Se refiere al conjunto de procedimientos previos que se realiza antes de la toma de la impresión definitiva de la boca con la finalidad de mejorar el diseño y la función de la PPR. Para que el diseño funcione satisfactoriamente, la boca del paciente, y en especial los dientes, deben ser modificados de manera que se pueda conseguir una forma más propicia para recibir a la futura PPR ${ }^{(5)}$.
Se debe diseñar colocando de manera estratégica los componentes de una PPR para controlar los movimientos de la prótesis que se originan durante la fase de masticación ${ }^{(10)}$.

Un diseño adecuado incluye a los conectores mayores rígidos que permitan una correcta distribución de fuerzas, retenedores indirectos estratégicamente ubicados, retenedores directos sobre los dientes pilares ${ }^{(11,12)}$. Un incorrecto diseño de una PPR puede ocasionar alteraciones severas en los tejidos bucales, problemas periodontales, entre otros ${ }^{(8)}$.

El laboratorio dental debe recibir una información correcta del diseño de la PPR ${ }^{(13-}$ ${ }^{15)}$, además es labor del odontólogo informar al paciente de la longevidad de la prótesis y de sus cuidados ${ }^{(14,16-18)}$. Existe una preocupación acerca de la habilidad y conocimientos de estudiantes de odontología de los últimos años y recién graduados en el diseño de PPR, por lo que surge la necesidad de una continua evaluación del aprendizaje en los estudiantes $(13,19-21)$

Diversas investigaciones realizadas en el Perú y en otros países muestran que estudiantes de los últimos años de las universidades desconocen el diseño adecuado de una PPR (22). Se han realizado estudios mostrando el nivel de conocimiento teórico y práctico sobre diseño de PPR ${ }^{(23,21)}$, la preparación de la boca (4) entre otros, determinando que la mayoría de estudiantes desconoce el diseño de la PPR.

Los resultados demostraron que no existe uniformidad de criterios para diseñar una prótesis, es por ello que surge la necesidad de realizar estudios que elaboren programas de capacitación en diseño de PPR en los estudiantes, docentes (24), comparación de metodologías de enseñanza para la asignatura de PPR $(25,26)$ etc. Por todo lo antes mencionado, el objetivo del presente estudio fue determinar la efectividad de un programa de capacitación en diseño de prótesis parcial removible en estudiantes de la asignatura de Internado Estomatológico de la Facultad de Odontología de la Universidad de San Martín de Porres. 


\section{MATERIALES Y MÉTODOS}

La muestra fue de tipo no probabilístico por conveniencia y estuvo conformada por todos los estudiantes matriculados en la asignatura de Internado Estomatológico del semestre 2019-I que cumplieron con los criterios de inclusión y exclusión. El estudio se realizó en enero y febrero del año 2019, participaron 70 estudiantes que cursaron por primera y única vez de manera regular y continua las asignaturas de PPR, Clínica Estomatológica I y II e Internado Estomatológico. Además, fueron informados de los procedimientos durante el desarrollo de la investigación y firmaron un consentimiento informado. El estudio fue de tipo pre-experimental, analítico, prospectivo y longitudinal.

Validación de los instrumentos (Juicio de Expertos): La validación de contenido fue por medio de un juicio de expertos en el que participaron 8 profesionales cirujanos dentistas con experiencia. Se aplicó la prueba del coeficiente de $V$ de $A_{i k e n}{ }^{27,28}$. La valoración por expertos se determinó en las diferentes áreas del instrumento preguntando ítem por ítem. Se consideró aceptable el ítem que tenga un consenso mayor a 0,75 .

Validación de los instrumentos (Estudio piloto): Se realizó un estudio piloto que estuvo conformado por 10 estudiantes de la asignatura de internado estomatológico de la FO-USMP pertenecientes al semestre 2018-II. Desarrollo del programa de capacitación en diseño de PPR: Se desarrolló en la Clínica Especializada de la FO-USMP. Tuvo un tiempo de duración de 4 horas, El objetivo del programa de capacitación fue consolidar los conocimientos teóricos y prácticos de los estudiantes en diseño de la PPR, aplicando estrategias y recursos didácticos para su entendimiento, entrenamiento y comprensión.

Se formaron pequeños grupos de estudiantes, se desarrolló la parte teórica y práctica, en donde se realizó la discusión y desarrollo de casos clínicos. Los casos clínicos estuvieron orientados a la discusión y desarrollo de un juicio clínico, pensamiento crítico por parte de los estudiantes, además ser capaces de planificar y diseñar la PPR. Se consiguió un estilo de aprendizaje activo y reflexivo para el estudiante en donde se incentivó el trabajo en equipo. El maestro tuvo como función inspirar, involucrar y motivar al estudiante durante toda la sesión de aprendizaje, fue un facilitador.

Se proporcionó a los estudiantes, modelos de estudio clase I y II de Kennedy (Figura 1), lápices de color rojo, azul y verde para diseñar los elementos de la base metálica y finalmente el color negro para marcar la extensión de la base de acrílico en los rebordes con extremo libre.

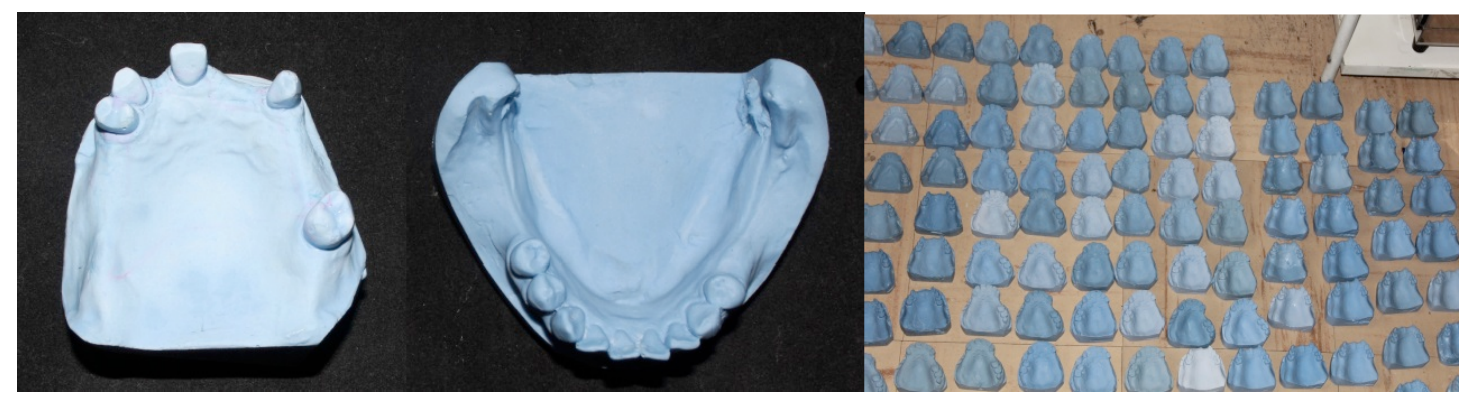

Figura 1. Modelos de Estudios Clase I y II de Kennedy para la evaluación procedimental de los estudiantes. Útiles para el diseño de la PPR 
Los instrumentos se midieron en 2 momentos: evaluación inicial y final, se desarrolló según los parámetros preestablecidos referidos en el estudio piloto para luego ser aplicados.

\section{"Evaluación inicial previa al programa de capacitación en diseño de PPR"}

Evaluación de la competencia Cognitiva: El instrumento para la evaluación cognitiva fue un cuestionario que estuvo conformado por 20 preguntas, se realizó en un tiempo de 40 minutos. Cada pregunta tuvo un valor de 1 punto, siendo la nota máxima 20 y la nota mínima 0.

Evaluación de la competencia Procedimental: Se entregó a cada estudiante 2 modelos clase I y II de acuerdo a la clasificación de Kennedy previamente paralelizados y con sus respectivos códigos de asignación. Se les entregó 3 lápices de colores y el tiempo para el desarrollo de la prueba fue de 1 hora. Finalizado los diseños, se mantuvieron cubiertos los códigos de asignación de cada modelo para cegar al evaluador respecto al nombre del participante y el momento en que fue realizado el diseño. Para la evaluación procedimental, se tomó en cuenta la Guía de Procedimientos para la Calificación del Diseño de $\mathrm{PPR}^{24}$ en donde la puntuación total máxima para cada clasificación de Kennedy | y || fue de 16 puntos.

"Evaluación final posterior al programa de capacitación en diseño de PPR"

Evaluación de la competencia Cognitiva: Se evaluó la competencia cognitiva mediante un Cuestionario, que estuvo compuesto de 20 preguntas y que tuvo un tiempo de 40 minutos. Cada pregunta tuvo un valor de 1 punto, siendo la nota máxima 20 y la nota mínima 0 .

Evaluación de la competencia Procedimental: Se entregó a cada estudiante 2 modelos clase I y II según la clasificación de Kennedy previamente paralelizados. El tiempo de duración fue de una hora. Se mantuvieron los códigos cubiertos para cegar al evaluador. Para la evaluación se tomó en cuenta La Guía de Procedimientos para la Calificación del Diseño de PPR ${ }^{(24)}$. La puntuación total máxima para cada clasificación de Kennedy I y II fue de 16 puntos.

Técnicas Estadísticas para el Procesamiento de la Información

Los datos obtenidos fueron analizados utilizando el paquete estadísticos IBM SPSS versión 25 así como también en el programa Excel 2010. Para garantizar que la técnica sea confiable, se empleó el coeficiente Alfa de Cronbach. La presentación de los resultados descriptivos fue mediante frecuencia $y$ porcentajes, las cuales fueron presentadas con tablas y gráficos. Para la prueba de hipótesis a probar, se aplicó el estadístico t-Student.

\section{Aspectos Éticos}

El trabajo de investigación tuvo la aprobación del Comité Revisor de Proyectos de Investigación (ACTA N026-2018) y por el Comité de Ética en Investigación (ACTA N006-2018-INVE/CEI-FO-USMP). Todos los estudiantes que participaron fueron informados de los procedimientos que se llevarían a cabo en el desarrollo de la investigación y firmaron un consentimiento informado. Se mantuvo en reserva la identidad del participante.

\section{RESULTADOS}

Se encontró que sólo el $42,85 \%$ de la muestra obtuvo nota aprobatoria para la evaluación de la competencia cognitiva inicial. Para la competencia procedimental inicial, en diseño de la clase I de Kennedy, se encontró que el $40 \%$ de la muestra obtuvo una nota desaprobatoria, de la misma forma en la evaluación del diseño de la clase II de Kennedy se encontró que el $60 \%$ de la muestra obtuvo nota desaprobatoria.

El análisis descriptivo de las evaluaciones cognitiva y procedimental en clase I y II de Kennedy antes y después de realizar el 
programa de capacitación en diseño de prótesis parcial removible evidenció diferencias significativas al inicio y al final de la capacitación (Ver Tabla 1).

Existe un incremento considerable en el promedio (media) de las evaluaciones iniciales y finales.
En el gráfico de caja y bigote se presentan los 3 grupos, en donde se observa las diferencias estadísticamente significativas $(p<0,001)$ entre las evaluaciones iniciales y finales de las competencias cognitivas y procedimentales. Nótese la distribución de los grupos en los momentos antes y después. (Ver Figura 2)

Tabla 1. Datos descriptivos generales de la evaluación cognitiva y procedimental en clase I y II de Kennedy antes y después de realizar el programa de capacitación en diseño de prótesis parcial removible.

\begin{tabular}{|c|c|c|c|c|c|c|c|}
\hline & \multirow[t]{2}{*}{ Media } & \multicolumn{2}{|c|}{$\begin{array}{l}95 \% \text { de intervalo de } \\
\text { confianza para la media }\end{array}$} & \multirow{2}{*}{ Mediana } & \multirow{2}{*}{$\begin{array}{l}\text { Desviación } \\
\text { Estándar }\end{array}$} & \multirow[t]{2}{*}{ Mínimo } & \multirow[t]{2}{*}{ Máximo } \\
\hline & & $\begin{array}{l}\text { Límite } \\
\text { inferior }\end{array}$ & $\begin{array}{l}\text { Límite } \\
\text { superior }\end{array}$ & & & & \\
\hline $\begin{array}{l}\text { Evaluación cognitiva } \\
\text { inicial }\end{array}$ & 10,81 & 10,02 & 11,60 & 10,00 & 3,32 & 4,00 & 18,00 \\
\hline $\begin{array}{l}\text { Evaluación cognitiva } \\
\text { final }\end{array}$ & 16,96 & 16,65 & 17,27 & 17,00 & 1,30 & 14,00 & 20,00 \\
\hline $\begin{array}{l}\text { Evaluación } \\
\text { procedimental inicial } \\
\text { en Clase I de Kennedy }\end{array}$ & 10,75 & 10,16 & 11,34 & 10,94 & 2,48 & 2,50 & 14,37 \\
\hline $\begin{array}{l}\text { Evaluación } \\
\text { procedimental final en } \\
\text { Clase I de Kennedy }\end{array}$ & 16,86 & 16,59 & 17,14 & 16,87 & 1,14 & 12,50 & 19,37 \\
\hline $\begin{array}{l}\text { Evaluación } \\
\text { procedimental inicial } \\
\text { en Clase II de } \\
\text { Kennedy }\end{array}$ & 9,43 & 8,60 & 10,26 & 9,37 & 3,48 & 1,87 & 16,87 \\
\hline $\begin{array}{l}\text { Evaluación } \\
\text { procedimental final en } \\
\text { Clase II de Kennedy }\end{array}$ & 16,04 & 15,68 & 16,39 & 16,25 & 1,49 & 12,50 & 18,75 \\
\hline
\end{tabular}




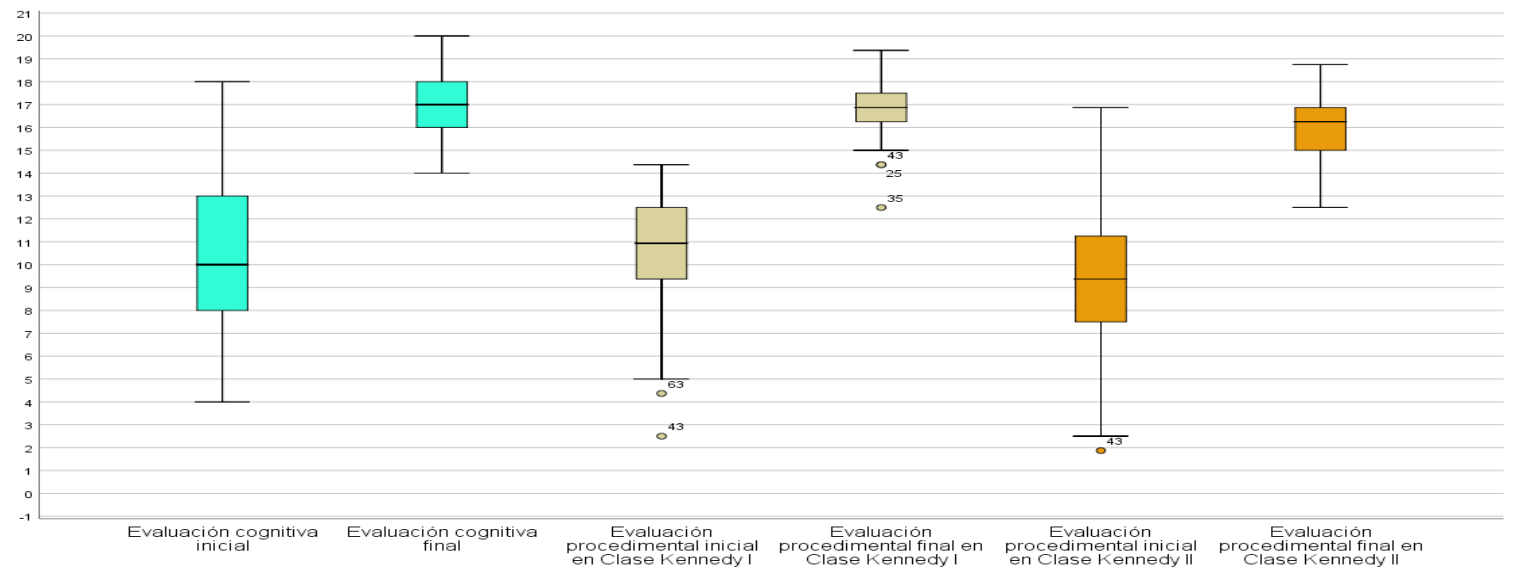

Figura 2. Gráfico general de distribución de todas las evaluaciones cognitivas y procedimentales en clase I y II de Kennedy antes y después de realizar el programa de capacitación en diseño de prótesis parcial removible.

La prueba de correlación no paramétrica Rho de Spearman evidencia que existe correlación estadísticamente significativa entre la evaluación cognitiva inicial y la evaluación procedimental inicial $(p<0,001)$. La magnitud de la correlación es considerable $(r=0,781)$. El correspondiente gráfico de dispersión de puntos muestra una distribución lineal ascendente entre ambas evaluaciones, lo cual refiere una evidente correlación directa. (Ver Figura 3) Por otra parte, la prueba de correlación no paramétrica Rho de Spearman muestra que existe correlación estadísticamente significativa entre la evaluación cognitiva final y la evaluación procedimental final $(p<0,001)$. Se observa una magnitud positiva débil en la correlación $(r=0,380)$. El correspondiente gráfico de dispersión de puntos muestra una distribución lineal ascendente estadísticamente significativa y de magnitud débil entre ambas evaluaciones, lo cual refiere la correlación directa (Ver figura 4).

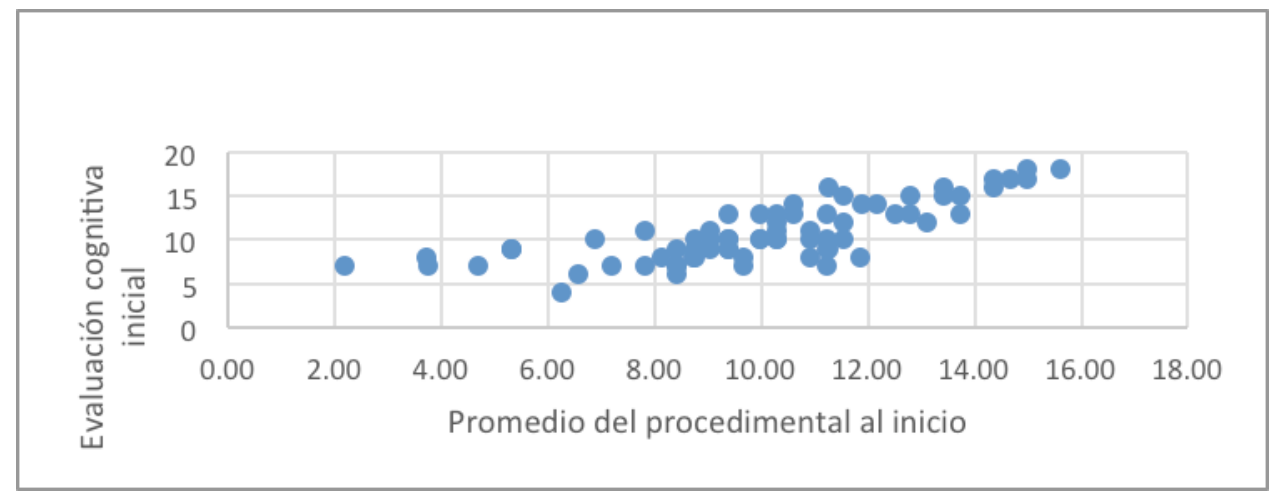

Figura 3. Dispersión de puntos que muestra la correlación entre la evaluación cognitiva y procedimental antes del programa de capacitación en diseño de prótesis parcial removible. 


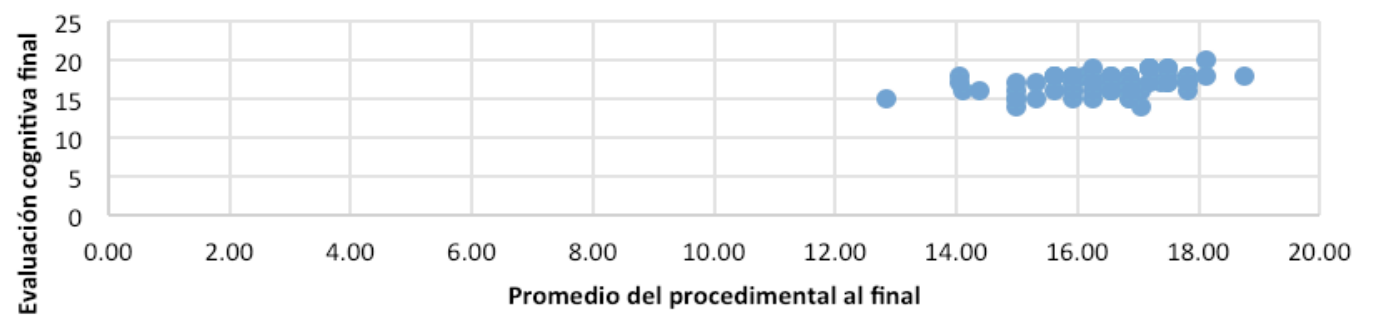

Figura 4. Dispersión de puntos que muestra la correlación entre la evaluación cognitiva y procedimental después del programa de capacitación en diseño de prótesis parcial removible.

\section{DISCUSIÓN}

El objetivo del estudio fue determinar la efectividad de un programa de capacitación en diseño de PPR en 70 estudiantes de la asignatura de Internado Estomatológico de la FO-USMP. Se evaluó al estudiante en una etapa inicial y final de la capacitación con la finalidad de comparar los resultados obtenidos. En la evaluación cognitiva inicial, se encontró que solo el $57,14 \%$ de la muestra obtuvo una nota aprobatoria, Khan MF et al (28). encontraron un resultado similar ya que mostraron en la evaluación de conocimientos que solo el $14 \%$ de los participantes tenían conocimientos en el diseño de PPR. Los resultados muestran que no hay uniformidad en los conceptos básicos teóricos en diseño de PPR en los estudiantes de las diferentes universidades del mundo.

En el presente estudio, la evaluación de la competencia procedimental inicial, para el diseño de la clase I de Kennedy, indicó que casi el $50 \%$ de la muestra obtuvo nota desaprobatoria, en clase II de Kennedy se encontró que el $60 \%$ de la muestra obtuvo nota desaprobatoria. Loza D ${ }^{(23)}$. mostró puntajes en la evaluación basal por debajo del $50 \%$ del valor máximo asignado a cada clase, además se debe mencionar que realizó su estudio en docentes del área de PPR. Los resultados indicaron que ningún estudiante realizó correctamente el diseño de la PPR. Mohamed TJ ${ }^{(29)}$, comparó los diseños de PPR según la experiencia de protesistas, estudiantes, odontólogos generales y técnicos dentales. Los protesistas obtuvieron mayor puntaje, seguidos por estudiantes de odontología.

En la presente investigación, sólo se incluyeron para la evaluación procedimental, las clases I y II de Kennedy, ya que ambas clases de Kennedy engloban todos los parámetros y criterios para realizar un diseño. Posterior a la capacitación en diseño de PPR, se evidenció para la competencia cognitiva, que todos los estudiantes obtuvieron nota aprobatoria, con un promedio de calificación de 16,96 de la misma forma, Loza $D^{(23)}$ obtuvo después de su capacitación teórica un logro cercano al puntaje máximo alcanzable. 
En la evaluación de la competencia procedimental en las Clase I y II de Kennedy todos los estudiantes obtuvieron una calificación aprobatoria, con promedios de 16. Los resultados también coinciden con el estudio de Loza $\mathrm{D}^{(23)}$ quien obtuvo puntajes superiores al $75 \%$ con respecto al puntaje máximo alcanzable para todas las clasificaciones. Echeto LF et al. ${ }^{(24)}$ y Echeto $\mathrm{LF}^{(25)}$ compararon los diseños de los estudiantes utilizando 2 metodologías, la primera tradicional y la segunda metodología TBL (Aprendizaje Basado en Equipos) obteniendo resultados favorables con el TBL, además de reflejar un cambio en las actitudes y conocimiento adquirido durante la metodología TBL. El aprendizaje integral del estudiante no solo involucra las competencias, sino también la metodología de enseñanza utilizada.

Se mostró una correlación estadísticamente significativa entre la evaluación cognitiva inicial y la evaluación procedimental inicial $(p<0,001)$. La magnitud de la correlación es considerable $(r=0,781)$, ello indica que los estudiantes que obtuvieron una calificación baja en la evaluación cognitiva, también obtuvieron calificaciones bajas en la evaluación procedimental. Los estudiantes que obtuvieron una calificación alta en la evaluación cognitiva, también obtuvieron calificaciones altas en la evaluación procedimental de ambas clases I y II.

Se demostró también que existe una correlación estadísticamente significativa entre la evaluación cognitiva final y la evaluación procedimental final $(p<0,001)$. Se observó una magnitud positiva débil en la correlación $(r=0,380)$. De igual forma Loza $D^{(23)}$ muestra resultados muy similares, por ello se interpreta que los estudiantes que obtuvieron una puntuación alta en la evaluación cognitiva, también obtuvieron mayor cantidad de componentes que diseñaron correctamente. Es importante mencionar que se debe delegar la responsabilidad del diseño de la PPR al odontólogo. Reem Haj-A et al. ${ }^{(30)}$ en su estudio muestran que el $89,9 \%$ de los trabajos recepcionados no brindan una explicación detallada del diseño a elaborar por parte del odontólogo, es por ello que los investigadores resaltan la importancia de brindar una información adecuada de los diseños por parte de los odontólogos. Dicha comunicación es necesaria no sólo con el laboratorio dental, sino también con el paciente ${ }^{(15)}$.En términos generales al comparar el logro de competencias cognitivas y procedimentales obtenidas en la evaluación inicial y final, se muestran diferencias estadísticamente significativas $(p<0.001)$, de igual manera Loza D ${ }^{(23)}$ encuentra puntajes que son superiores al $75 \%$ con respecto al puntaje máximo alcanzable.

Finalmente, al comparar las evaluaciones cognitivas y procedimentales antes y después de realizar el programa de capacitación, se evidenció un nivel significativo de mejoramiento con respecto al diseño de PPR, es decir, el programa de capacitación obtuvo resultados favorables, demostrando la necesidad de realizar programas de capacitación de manera constante a los estudiantes de las distintas asignaturas de odontología.

Los resultados de la investigación son relevantes debido al aporte en el ámbito educacional, ya que brinda información importante para el equipo docente sobre el nivel en el que se encuentran los estudiantes. Los estudiantes demostraron falencias en ambas competencias tanto cognitivas como procedimentales en la fase inicial, lo que nos indicaría que dichas deficiencias se originaron durante la formación de su carrera odontológica.

Una capacitación constante para los estudiantes, egresados y docentes, permitirá que puedan conocer y actualizar los principios y fundamentos básicos en el diseño de la prótesis. Dentro de las limitaciones que se observaron fueron la falta de disponibilidad que muchos de los estudiantes mostraron debido al desinterés que mostraron. 
Otros estudiantes no pudieron participar por la poca disponibilidad en sus horarios debido a sus diferentes rotaciones en la clínica de la universidad. Se considera que el tamaño de muestra debió ser mayor para poder encontrar relaciones y generalizaciones mucho más significativas. El programa de capacitación debió tener una mayor duración, pero no se pudo por la disponibilidad de los estudiantes. No se registró abandono de los participantes durante el estudio.

Se concluye que existen diferencias estadísticamente significativas entre las evaluaciones cognitivas antes y después del Programa de capacitación en diseño de prótesis parcial removible, evidenciándose un nivel significativo de mejoramiento del diseño. Es por ello que se determinó la efectividad del programa de capacitación. A partir de los resultados favorables obtenidos en la investigación, se recomienda capacitaciones continuas para unificar criterios, de esta forma potencializar las metas de la asignatura de PPR.

Contribuciones de autoría: RCM, JOGC, RMV, NTT, JAF: Participaron en el diseño del estudio, revisión de la literatura, recolección de datos, análisis e interpretación de resultados redacción, revisión y aprobación del artículo.

Fuente de financiamiento: Autofinanciado.

Conflictos de interés: Los autores no tienen ningún conflicto de interés.

\section{REFERENCIAS BIBLIOGRÁFICAS}

1. Núñez $M$. Modelos educativos de postgrado: teoría y ejecución. Ciencia y Educación 2018; 2(3): 29-43.

2. Juliá, M. T. Competencias generales de la formación universitaria: aportes a la calidad con equidad". En G. Carrillo (Ed.), I Encuentro Internacional Universitario. El currículo por competencias en la Educación Superior. Ponencias y debate (pp. 39-62). Lima: Pontificia Universidad Católica del Perú. (2015).
3. Icaza JL, Ludeña MA, Bernabé E, BeltránNeira RJ. Auto-percepción del dominio de competencias clínicas odontológicas entre recién graduados de la Facultad de Estomatología de la Universidad Peruana Cayetano Heredia. Rev Estomatol Herediana. 2006;16(1):9-14.

4. Farias Neto A, Duarte A, Shiratori FK, De Alencar e Silva Leite $\mathrm{PH}$, Rizzatti-Barbosa $\mathrm{CM}$, Cardoso W. Evaluation of senior Brazilian dental students about mouth preparation and removable partial denture design. J Dent Educ. 2010;74(11):125560.

5. Loza D, Valverde R. Diseño de Prótesis Parcial Removible. Lima: Editorial Ripano; 2006. 228 p.

6. Tregerman I, Renne W, Kelly A, Wilson D. Evaluation of removable partial denture frameworks fabricated using 3 different techniques. J Prosthodont. 2019;122(4):390-5.

7. Gad M, Abualsaud R, Al-Thobity A, AlAbidi A, Khan S, Abdel-Halim $M$ et al. Prevalence of partial edentulism and RPD design in patients treated at College of Dentistry, Imam Abdulrahman Bin Faisal University, Saudi Arabia. J Saudi Dental. 2020; 72:74-79.

8. Tada S, Allen PF, lkebe K, Matsuda K, Maeda Y. Impact of periodontal maintenance on tooth survival in patients with removable partial dentures. J Clin Periodontol 2015; 42:46-53.

9. Le Bars P, Ayepa A, Koffi J, Badran Z, Soueidan A. Relationship between removable prosthesis and some systemic disorders. J Indian Prosthod Soc. 2015; 15:292-9.

10. Mahrous A, Schneider G. Enhancing Student Learning of Removable Prosthodontics Using the Latest Advancements in Virtual 3D Modeling. J Prosthodont. 2019;28:471-2.

11. Kim J. Revisiting the Removable Partial Denture. Dent Clin North Am. 2019; 63(2):263-278.

12. Alageel $\mathrm{O}$, Ashraf $\mathrm{N}$, Bessadet $\mathrm{M}$, Nicolas $E$, Tamimi $F$. Evaluation of the design driven prediction of removable par denture retention. $\mathrm{J}$ Prosthet Dent. 2019;(19):30667-5. 
13. Arbab S, Khalifa N, Nasser $M$. Communication Between Dentists and Dental Technicians During the Fabrication of Removable Partial Dentures in Khartoum State, Sudan. Acta stomatol Croat. 2018;52(3):246-253.

14. Özyemişci N. Factors associated with insufficient removable partial denture design instructions. Dent Med Probl. 2018;55(2):173-177.

15. Kujtim Sh,Dula L, Pustina-Krasniqi T, Bicaj $T$, Ahmedi F, Lila-Krasniqi $Z$ et al. Patient's Satisfaction with Removable Partial Dentures: A Retrospective Case Series. J Open Dent, 2016;10: 656-663.

16. Bhola S, Hellyer P, Radford D. The importance of communication in the construction of partial dentures. $\mathrm{J} \mathrm{Br}$ Dent. 2018;224(11):853-6.

17. Polyzois G, Lagouvardos P, Kranjcic J, Vojvodic D. Flexible Removable Partial Denture Prosthesis: A Survey of Dentists' Attitudes and Knowledge in Greece and Croatia. Act Stomatol Croat. 2015;49(4):316-24.

18. Moreno A, Haddad M, Coelho M, Rocha E, Assuncao W, Filho $\mathrm{H}$ et al. Epidemiological Data and Survival Rate of Removable Partial Dentures. J. Clin. Diagnostic Res. 2016;10(5): 84-7.

19. Lynch $C D$, Singhrao $H$, Addy LD, Gilmour AS.The teaching of fixed partial dentures in undergraduate dental schools in Ireland and the United Kingdom.J Oral Rehabil. 2010;3(37):908-15.

20. Mather $H$, Thomason $M$, Ellis J. Are UK graduates equipped with the skill set required to meet the demands of the UK's edentulous population?. J Br. Dent .2018;(225):1-4.

21. Brand HS, Kamell H, Kharbanda AK, Dozic A. Students' Perceptions of Materials and Techniques Used at European Dental Schools in the Education of Fixed Prosthodontics. J Dent Educ. 2013;107(9):1140-6.

22. Revoredo de Rojas A. Nivel de conocimientos y factores críticos autopercibidos en la enseñanza del diseño de

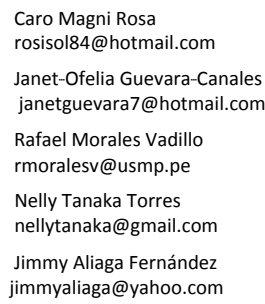

prótesis parcial removible por alumnos del quinto año de Estomatología. Rev Estomatol Herediana. 2007; 17:29-34.

23. Bonnet G, Lance C, Bessadet M, Tamini F, Veyrune JL, Francois $\mathrm{O}$, et al. Teaching removable partial denture design: 'METACIEL, a novel digital procedure. Int J Public Health.2018;9:24-5.

24. Loza D. Identificación de factores críticos en el diseño de la prótesis parcial removible a través de la evaluación de un programa de capacitación aplicado a docentes de la Facultad de Estomatología de la Universidad Peruana Cayetano Heredia. [Tesis para Optar el Grado de Doctor en Estomatología]. Lima: Universidad Cayetano Heredia; 1999.

25. Echeto LF, Sposetti V, Childs G, Aguilar $\mathrm{ML}$, Behar-Horenstein LS, Rueda L, et al. Evaluation of Team-Based Learning and Traditional Instruction in Teaching Removable Partial Denture Concepts.J Dent Educ.2015; 79(9):1040-8.

26. Echeto LF. Removable partial denture components and applications: a teambased learning module. MedEdPORTAL. 2016;12:1-5.

27. Soto CM. Intervalos de confianza asimétricos para el índice la validez de contenido: Un programa Visual Basic para la $\mathrm{V}$ de Aiken. Anales de psicología. 2009,25(1):159-61.

28. Aiken LR. Three coefficients for analyzing the reliability and validity of ratings. Educ Psychol Meas.1985;45(1):131-42.

29. Khan MF, Khan FNA, Lone MA, Hussain MW, Shaikh MA, Shaikh IA. Knowledge and attitude regarding designing removable partial denture among interns and dentist; dental schools in Pakistan. J Pak Dent Assoc 2020;29(2):66-70.

30. Mohamed TJ. The Assessment of Different Levels of Dental Participants on Designing a Removable Partial Denture. The Ass of Diff Levels Dent. 2016;13(1):57-63.

31. Reem Haj A,Quran F, Adel O. Dental Laboratory Communication Regarding Removable Dental Prosthesis Design in the UAE. J Prosthodont. 2012; 21(5):425-8.

\footnotetext{
https://orcid.org/0000-0002-5822-9305

http://orcid.org/0000-0002-7040-8269

https://orcid.org/0000-0002-7835-6408

Dhttps://orcid.org/0000-0003-4742-4110

httpd://orcid.org/ 0000-0001-6496-9927
} 